\title{
La transmission de signal utilisant les seconds messagers
}

\author{
C. Pfister
}

Centre d'études nucléaires de Grenoble, biophysique moléculaire et cellulaire, BP $85 X$, 38041 Grenoble Cedex, France

(14éunion du groupe Développement INRA, Clermont-Ferrand, 25-27 mai 1988)

Résumé - La communication intercellulaire utilise des signaux moléculaires (hormones, neurotransmetteurs, facteurs de croissance) émis par une cellule et reconnus par des cibles. Un mécanisme classique d'action de ces molécules fait intervenir un récepteur membranaire, une protéine de couplage GTP-dépendante, un effecteur qui module la concentration intracellulaire d'un second messager (AMPc, GMPc, inositol trisphosphate, $\mathrm{Ca}^{++}$), et une cible pour l'effet final de ce second messager (kinases, canal). Les systèmes hormonaux et la phototransduction dans les segments externes de bâtonnets de vertébrés sont les exemples les plus connus de ce mécanisme. Ils servent ici d'illustration de l'état des connaissances en ce qui concerne les interactions entre les protagonistes des cascades enzymatiques de transduction.

hormones - phototransduction — protéines G — adénylate cyclase — aluminofluorures

Summary - Signal transmission using second messengers. Intercellular communication needs signal molecules (hormones, neurotransmitters, growth factors) emitted by a cell and recognized by targets. A widespread mechanism of cellular action of these molecules involves a membrane receptor, a GTP-dependent coupling protein, an effector which modulates the intracellular concentration of a second messenger (CAMP, cGMP, inositol triphosphate, $\mathrm{Ca}^{++}$), and a target for final effect of this second messenger (kinases, channel). Hormonal systems and visual processes in vertebrates rod outer segments are the best known examples of this mechanism; they are taken here to illustrate the state of knowledge of the interactions between the actors of the transductional enzymatic cascades.

hormones - phototransduction - G-proteins - adenylate cyclase - fluoroaluminate 


\section{LA TRANSDUCTION MEMBRANAIRE}

\section{Introduction}

Dans un organisme multicellulaire, des voies de communication diverses permettent aux cellules de réguler leur division, leur organisation en tissus, ou de coordonner leurs diverses activités (métabolisme, contraction, sécrétion, etc.). La communication chimique est une voie particulièrement importante : une cellule émettrice libère dans le milieu extérieur un signal chimique (médiateur chimique local, hormone ou neurotransmetteur, facteur de croissance), détecté ensuite par une cellule réceptrice (cellule cible). Dans chaque cellule cible, ces médiateurs chimiques sont reconnus par un récepteur protéique, et initient ainsi la transformation (la transduction) du signal en réponse cellulaire.

Certains médiateurs chimiques, en particulier certaines hormones, ont un caractère hydrophobe et pénètrent dans la cellule par simple diffusion à travers la membrane lipidique, et s'associent alors à des récepteurs protéiques cytoplasmiques. Les médiateurs chimiques hydrophiles, eux, sont arrêtés par la membrane, et sont détectés par des récepteurs intégrés dans cette membrane. C'est de ce deuxième type de transduction membranaire qu'il sera discuté ici.

Les modes de transduction membranaires, peuvent être classés en 4 catégories (Tableau I) :

1) le récepteur est un canal : c'est le cas des récepteurs à l'acétylcholine ou à l'acide gamma-amino-butyrique (GABA) : leur conductance augmente par interaction avec le ligand. C'est également le cas de la bactériorhodopsine : la photo-isomérisation du rétinal, photopigment de ce récepteur, induit une conductivité aux protons à travers la protéine. Les récepteurs sont formés de plusieurs hélices $\alpha$ transmembranaires. Le temps de réponse est très court (de l'ordre de la milliseconde);

2) le récepteur possède une fonction enzymatique propre : par exemple, le récepteur du facteur de croissance épidermique (EGF) ou de l'insuline, sur lequel la liaison de l'hormone stimule une fonction d'autophosphorylation de tyrosines. Dans ce cas, le récepteur traverse la membrane par une seule hélice $\alpha$ transmembranaire. Les temps de réponse sont beaucoup plus longs, et peuvent atteindre plusieurs minutes;

3) le récepteur excité déclenche une cascade enzymatique : par l'intermédiaire d'une uprotéine de couplage GTP. dépendante" ou "protéine G», un effecteur enzymatique intracellulaire est stimulé, ce qui in fine conduit à la modulation de concentration d'une espèce soluble ou "second messager". Les temps de réponse de ces systèmes sont relativement variables, en général moyennement rapides (secondes ou minutes) sauf dans le cas particulier de la vision. Ce type de mécanisme, très largement répandu, a fait l'objet de multiples publications récentes (Stryer \& Bourne, 1986; Gilman, 1987; Dolphin, 1987; Spiegel, 1987; lyengar \& Birnbaumer, 1987; Miller, 1988; Neer \& Clapham, 1988). II est schématisé sur la Figure 1 et nous en détaillerons les mécanismes sur 2 exemples;

4) la protéine de couplage GTPdépendante, activée par interaction avec le récepteur lié a un effet direct sur un canal, dont la conductivité est alors modulée.

\section{Exemples de transduction membranaire à second messager}

Parmi tous les systèmes connus dans cette catégorie, 2 occupent une place de choix et servent de référence. 
Tableau I. Exemples de modes de transduction

\begin{tabular}{|c|c|c|c|c|c|c|}
\hline & $\begin{array}{l}\text { Types de } \\
\text { récepteurs }\end{array}$ & Molécules signa & Effecteurs & Signal cellulaire & $\begin{array}{l}\text { Temps de } \\
\text { réponse }\end{array}$ & \\
\hline 1 & $\begin{array}{l}\text { Récepteur } \\
\text { nicotonique }\end{array}$ & $\begin{array}{l}\text { Acétylcholine } \\
\text { GABA }\end{array}$ & $\begin{array}{l}\text { Le récepteur } \\
\text { est un canal }\end{array}$ & $\begin{array}{l}\text { Influx d'ions } \mathrm{Na}^{+} \text {et } \\
\mathrm{Ca}^{++} \text {et dépolarisation } \\
\text { de la membrane }\end{array}$ & $\mathrm{ms}$ & \\
\hline \multicolumn{2}{|c|}{ Bactériorhodopsine } & & & Flux de $\mathrm{H}^{+}$ & & \\
\hline II & $\begin{array}{l}\text { Récepteur à } \\
\text { I'EGF }\end{array}$ & EGF & $\begin{array}{l}\text { Le récepteur } \\
\text { est une } \\
\text { Tyr-kinase }\end{array}$ & $\begin{array}{l}\text { Autophosphorylation du } \\
\text { récepteur, el phosphorylation } \\
\text { d'autres protéines }\end{array}$ & $\min$ & \\
\hline \multirow[t]{3}{*}{ III } & $\begin{array}{c}\text { Récepteur } \\
\beta \text { adrénergique }\end{array}$ & Adrénaline & $\begin{array}{l}\text { Adénylate } \\
\text { cyclase }\end{array}$ & Synthèse d'AMPc & $s-\min$ & \\
\hline & $\begin{array}{l}\text { Récepteur } \\
\text { aux IgE }\end{array}$ & $\lg E$ & Phospholipase C & Production de IP3 et DAG & $s-\min$ & $\begin{array}{l}\text { Protéine } \\
\qquad\end{array}$ \\
\hline & Rhodopsine & Lumière & Phosphodiestérase & Hydrolyse de GMPC & $100 \mathrm{~ms}$ & \\
\hline IV & Récepteur & Acétyicholine & Canal $\mathrm{K}^{+}$ & Efflux de $\mathrm{K}^{+}$ & & \\
\hline
\end{tabular}

\section{Le système de l'adénylate cyclase}

L'AMPcylique (AMPc) a été le premier médiateur intracellulaire identifié. II est impliqué dans de nombreuses réponses hormonales et neurohormonales. II agit par intervention d'enzymes spécifiques, les protéines kinases AMPc-dépendantes, qui sont responsables de la phosphorylation, et donc de la régulation d'activité, de certaines protéines qui, à leur tour, contrôlent la réponse cellulaire.

L'adénylate cyclase est l'enzyme responsable de la synthèse de l'AMPc à partir d'ATP. Présente dans de nombreux tissus, son activité est stimulée ou inhibée par diverses hormones, chacune spécifique d'un type cellulaire. L'activité enzymatique est

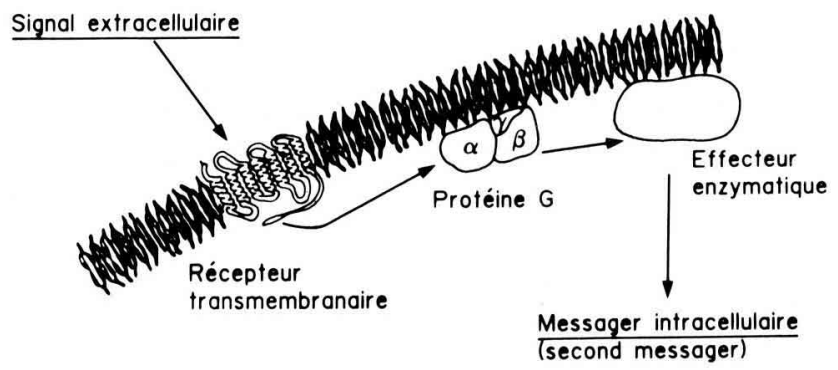

Fig. 1. Schéma gènèral des systèmes de transduction membranaire impliquant une protêine $G$. 
portée par une protéine distincte des récepteurs (Limbird \& Lefkowitz, 1977). Les récepteurs sont répartis en 2 classes : ceux qui induisent une stimulation de l'adénylate cyclase, et ceux qui induisent une inhibition de l'enzyme. Dans le système stimulé par le glucagon, Rodbell et al. (1971) ont montré que l'activation de l'adénylate cyclase était dépendante de la présence de GTP, et Pfeuffer (1977) a montré que 2 protéines étaient impliquées, l'une portant l'activité cyclase, et l'autre liant le GTP (“protéine G»). C'était le premier exemple d'une cascade enzymatique récepteur-protéine G-effecteur.

\section{Le système de la phototransduction dans les bâtonnets rétiniens}

Le bâtonnet (Fig. 2A) est une cellule capable de répondre à la stimulation par un seul photon. L'hyperpolarisation de la membrane plasmique qui en résulte provient de la fermeture de canaux cationiques de cette membrane, dont la conductance est directement contrôlée par la concentration intracellulaire de GMP cyclique (Fesenko et al., 1985; Owen, 1986).

La morphologie très particulière de cette cellule en rend l'étude remarquablement féconde : toutes les protéines responsables de la phototransduction sont rassemblées dans le segment externe du bâtonnet, alors que les machineries énergétique (mitochondries) et génétique (noyau), etc. sont rassemblées dans le segment interne. Ces 2 parties de la cellule se séparent aisément, au niveau du cil connectif, par simple agitation des rétines dans un tampon aqueux, et on obtient, après une étape de purification sur gradient de saccharose, une préparation "pure", en ce sens qu'elle contient exclusivement les protéines intéressant le phénomène de phototransduction : le récep- teur (la rhodopsine), la protéine G (la transducine), l'effecteur (la phosphodiestérase à GMP cyclique) et le canal cationique de la membrane plasmique. II faut souligner de plus que, à l'exception du récepteur et du canal cationique, les protéines intéressantes telles que la transducine et la phosphodiestérase (ainsi qu'un certain nombre d'autres protéines dont nous ne parlerons pas ici) sont des protéines solubles ou périmembranaires (Fig. 2B); ceci a permis de les isoler (Kühn, 1980), de les caractériser, d'en étudier les fonctions, en s'affranchissant de tout problème de dénaturation par les détergents.

Le système de la phototransduction est pris, tant dans la littérature (Chabre et al., 1984; Stryer \& Bourne, 1986) que dans
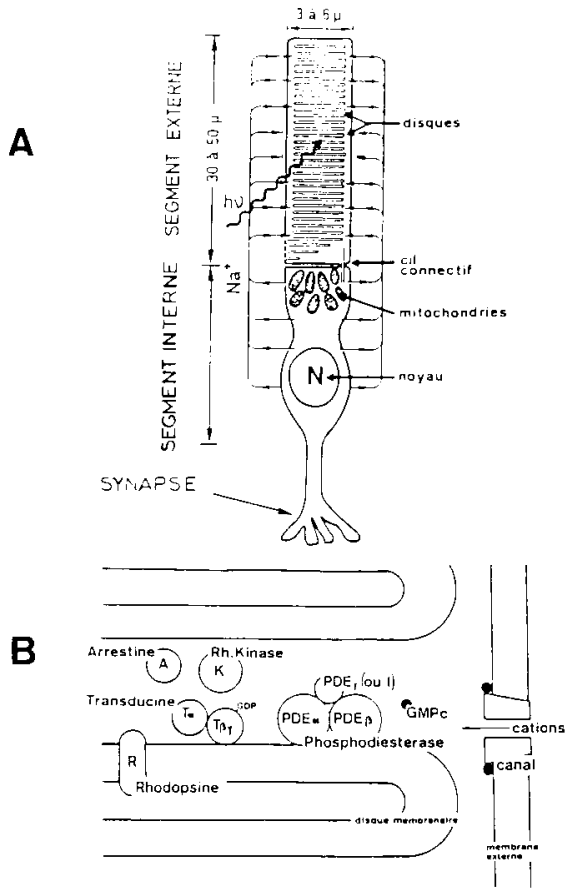

Fig. 2. Représentation schématique d'un bâtonnet $(A)$, et de l'organisation des protéines dans la membrane des disques ou dans l'espace interdiscal (B). 
cet exposé, comme archétype de transduction membranaire à second messager.

\section{LES RÉCEPTEURS}

\section{La rhodopsine}

Elle est constituée d'un polypeptide transmembranaire, l'opsine (39 kDa) (Fig. 3) et d'un chromophore, le rétinal (dans sa conformation 11-cis à l'état de repos). De nombreuses études biophysiques et biochimiques (pour revue, voir Chabre, 1985) ont permis d'élaborer un modèle structural de $R$ qui s'accorde avec la séquence primaire de l'opsine déterminée ultérieurement (Hargrave et al., 1983; Ovchinnikov, 1982). Sept hélices $\alpha$ hydrophobes constituent le cœur transmembranaire de la protéine, et sont reliées par des boucles cyto- plasmiques et intradiscales, formant 2 domaines hydrophiles de part et d'autre de la membrane. II faut souligner que l'arrangement dans l'espace de ces 7 hélices $\alpha$, tel que représenté sur la Figure 3, n'est qu'une généralisation d'observations effectuées sur la bactériorhodopsine. Dans la membrane pourpre, en effet, cette protéine possède un arrangement quasi cristallin, qui a permis des études en diffraction de rayon $X$; l'existence de 7 zones de forte densité électronique a été corrélée à la présence, dans la structure primaire de la protéine, de 7 régions de forte hydrophobicité, très probablement arrangées en hélices $\alpha$. Dans la rhodopsine, le rétinal est lié par une liaison de type "base de Schiff" à la lysine 296, à peu près au centre de la septième hélice, où il se trouve aligné dans un plan très proche de celui de le membrane. Remarquons que seulement $25 \%$ de la masse protéique (l'équivalent d'une protéine d'environ $10 \mathrm{kDa}$ ) se trouve exposé vers la face cytoplasmique du disque). cytoplasme

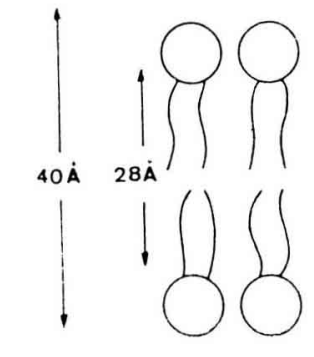

intērieur des disques

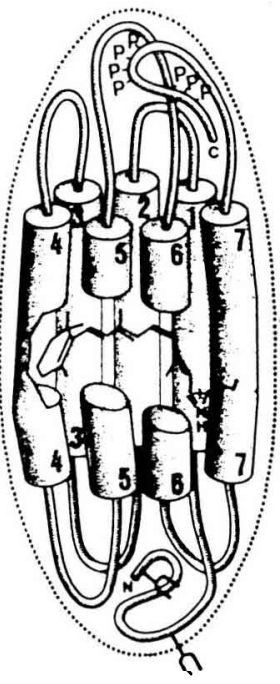

pôle cytoplasmique:

$25 \%$ de la masse

site liaison transducine

phosphate / liaison arrestine

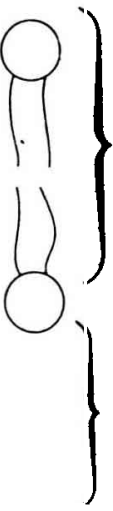

Cœur hydrophobe

7 hélices $\alpha$

$50 \%$ de la masse

site du rétinal

pôle intradiscal

$25 \%$ de la masse

carbohydrates

Fig. 3. Représentation schématique de la rhodopsine. 


\section{Les récepteurs hormonaux}

Les séquences primaires des récepteurs hormonaux $\beta$-adrénergiques ( $\beta 1$ et $\beta 2$ ), muscariniques cholinergiques (M1 et M2) et de la rhodopsine ont été comparées (Dohlman et al., 1987). Peu d'homologies strictes de séquences existent entre ces récepteurs, mais tous semblent constitués de 7 hélices $\alpha$ hydrophobes transmembranaires, reliées par des boucles hydrophiles sur chaque face de la membrane. Dans la rhodopsine, le rétinal est considéré comme l'équivalent d'une hormone silencieuse, précouplée à son récepteur. Dans les hélices $\alpha$ des récepteurs hormonaux, des résidus chargés particulièrement conservés occupent des positions correspondant aux sites d'interaction du rétinal dans $R$, et interviendraient dans la liaison de l'hormone à son récepteur (Applebury \& Hargrave, 1986); ces résultats, confirmés par des expériences de mutagenèse dirigée, montrent que l'hormone, bien qu'hydrophile, se lie dans la zone hydrophobe du récepteur (Dixon et al., 1987; Strader et al., 1988).

\section{La photoactivation de la rhodopsine}

Le 11-cis rétinal, absorbant un photon, s'isomérise en 11-trans rétinal, provoquant une série de transconformations de la protéine pouvant aller jusqu'à la dissociation de l'opsine et du rétinal. L'apparition de divers intermédiaires structuraux de la rhodopsine, leur caractérisation spectrale, ainsi que leur cinétique d'évolution sont connus (Chabre, 1985). Nous ne retiendrons ici qu'un seul de ces intermédiaires, l'état Meta II (absorption à $380 \mathrm{~nm}$ ) qui a été caractérisé comme étant la forme active de $\mathrm{R}$ dans la cascade enzymatique de la phototransduction (Bennett et al., 1982).
C'est l'état que nous symboliserons par la suite par $\mathrm{R}^{\star}$.

\section{LES PROTÉINES DE COUPLAGE GTP. DÉPENDANTES}

L'étude de la transducine $(T)$ et des protéines $G$ (notées ici $G$ ) a permis de mettre en évidence quelques caractéristiques de ces protéines (Stryer \& Bourne, 1986).

Toutes sont composées de 3 polypeptides : $\alpha, \beta$ et $\gamma$ de poids moléculaires similaires (Tableau II); un site nucléotidique est localisé sur la sous-unité $\alpha$. Cette même sous-unité porte (le plus souvent) un site d'ADP-ribosylation par les toxines du choléra (CTX) ou de Bordetella pertussis (PTX).

Dans les séquences primaires d'un certain nombre des sous-unités $\mathrm{G} \alpha$ se retrouve une alternance de régions particulièrement conservées (50 à $90 \%$ d'homologies) et de régions très divergentes. A chacune de ces régions, les auteurs attribuent des fonctions bien précises telles que liaison du nucléotide, interaction avec le récepteur, interaction avec l'effecteur, ou avec les sous-unités $\mathrm{G} \beta \gamma$, spéculations quelquefois controversées.

Les sous-unités $\beta$ et $\gamma$ forment un complexe, indissociable dans des conditions non dénaturantes. Ces complexes semblent fonctionnellement identiques dans tous les systèmes, et peuvent même être interchangés. Les polypeptides $\beta$ purifiés sont composés des mêmes acides aminés mais $\mathrm{G} \beta$ se présente sous la forme d'un doublet ( 35 et $36 \mathrm{kDa}$ ); ces 2 formes ont la même capacité d'inhibition sur l'activité cyclase, mais sont reconnus sélectivement par différents anticorps. La transducine ne présente qu'une forme ( $36 \mathrm{kDa}$ ). 
Tableau II. Comparaison entre protéines $\mathrm{G}$ de divers systèmes de transduction

\begin{tabular}{|c|c|c|c|c|c|}
\hline Système & $\begin{array}{l}\text { Composants } \\
\text { de la } \\
\text { protéine }\end{array}$ & $\begin{array}{c}P M \\
\text { (selon } \\
\text { gel SDS) } \\
(k D a)\end{array}$ & $\begin{array}{c}P M \\
\text { (selon } \\
\text { séquence } \\
(k D a)\end{array}$ & Toxine & Références \\
\hline Vision & $\begin{array}{l}\text { Transducine : } \\
\qquad \begin{array}{c}\alpha_{1} \\
\beta \\
\gamma \\
\alpha_{2}\end{array}\end{array}$ & $\begin{array}{r}39 \\
36 \\
8 \\
39\end{array}$ & $\begin{array}{r}40 \\
37,4 \\
8,4 \\
40,4\end{array}$ & $\begin{array}{l}\text { CTx, PTx } \\
?\end{array}$ & $\begin{array}{l}\text { 1: Stryer \& Bourne (1986) } \\
\text { 2: Proud (1986) }\end{array}$ \\
\hline Cyclase & Gs : $\begin{array}{c}\alpha \text { s } \\
\beta \\
\gamma\end{array}$ & $\begin{array}{c}52 / 45 \\
35 / 36 \\
8\end{array}$ & $\begin{array}{c}46 / 44,5 \\
37,3 / 37,4\end{array}$ & CTx & 1-2-3: Gilman (1987) \\
\hline & $\begin{aligned} G i: \alpha i \\
\beta \\
\gamma\end{aligned}$ & $\begin{array}{c}41 / 40 \\
35 / 36 \\
8\end{array}$ & $\begin{array}{l}40,4 / 40,5 \\
37,3 / 37,4\end{array}$ & Ptx & 1-2-4: Graziano \& Gilman (1987) \\
\hline Inconnu & 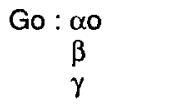 & $\begin{array}{c}39 \\
35 / 36 \\
8\end{array}$ & $\begin{array}{c}39,9 \\
37,3 / 37,4\end{array}$ & P.TX & 1 \\
\hline
\end{tabular}

Les sous-unités $\gamma$ des différentes protéines $\mathrm{G}$ étudiées ne sont pas discernables, associées indistinctement semblet-il à $\beta 35$ ou $\beta 36$. Seule T $\gamma$ est très différente.

\section{COUPLAGE RÉCEPTEUR-PROTÉINE G : ACTIVATION DE LA PROTÉINE G}

Les mécanismes d'un tel couplage ont été particulièrement décrits sur le système visuel (Stryer \& Bourne, 1986; Chabre, 1985; Chabre et al., 1988).

\section{Activation de la transducine}

Dans son état inactif (avant illumination, état 1 de la Figure 4A), la transducine est sous forme d'un complexe T $\alpha$-GDP-T $\beta \gamma$, et ce complexe est périmembranaire. Le site nucléotidique de $T \alpha$ n'est pas directement accessible aux nucléotides libres du milieu. Après photoactivation, $R^{*}$ interagit avec $T$ et provoque l'ouverture du site nucléotidique de T $\alpha$. Le GDP présent est alors libéré, et peut être remplacé par un GTP (Fig. $4 A$ et $4 B$, étapes 2 à 4 ). Une fois son site occupé par un GTP, T $\alpha$ se dissocie de $R^{*}$ et de $T \beta \gamma$, et devient soluble dans l'espace interdiscal (Fig. 4A et B, étape 5).

Trois points sont à souligner :

- T $\alpha$ dans son état de repos (T $\alpha$ GDP$T \beta \gamma$ ainsi que dans son état activé (T $\alpha$ GTP) porte un nucléotide non échangeable, dans un site non accessible. Ce site n'est "ouvert" que pendant la durée de vie du complexe $R^{*}-T$. C'est ce qui est symbolisé sur la Figure 4B. 

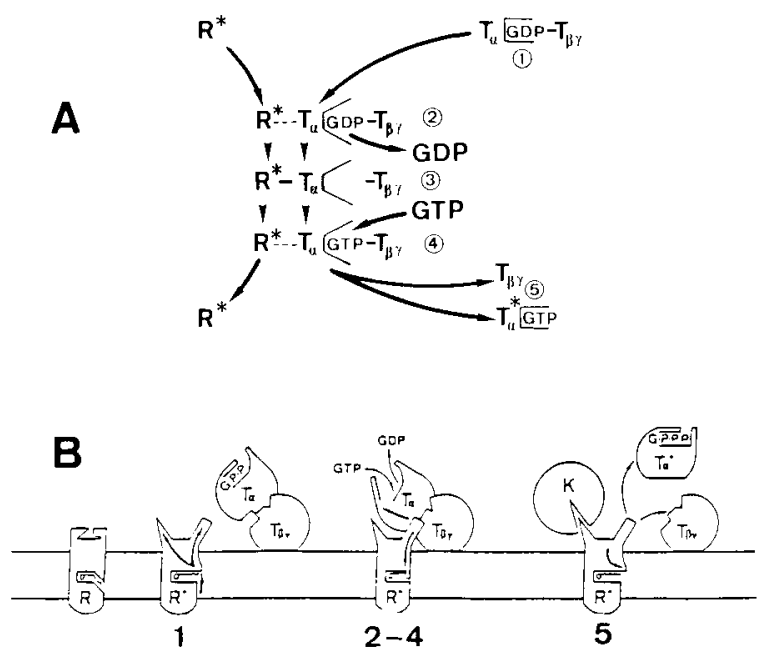

Fig. 4. Représentation schématique des diverses étapes de l'interaction rhodopsine/transducine et de l'échange GDP/GTP sur la transducine.

- il n'existe pas de "précouplage" fonctionnel entre la rhodopsine inactive et la transducine. Une seule $R^{\star}$ catalyse l'échange GDP/GTP sur plusieurs centaines de $T$, et la stœechiométrie $R / T$ est de $10 \mathrm{R}$ pour une seule $\mathrm{T}$.

La transducine ne présente aucune zone intramembranaire. C'est hors de la membrane que s'effectuent ses interactions avec la rhodopsine, dont la boucle entre les hélices 5 et 6 joue un rôle particulièrement important (Stryer \& Bourne, 1986).

\section{Le complexe $R^{*}$-Tvide}

Entre le relâchement du GDP (étape 2 de la Figure 4A) et la charge en GTP (étape 4 de la Figure 4A), il existe nécessairement un état intermédiaire dans lequel le site nucléotidique est "vide" (état 3 de la Figure 4A). Ce complexe $R^{\star}-$ Tvide peut être stabilisé si le milieu ne contient aucun nucléotide (ni GDP ni GTP), et ceci même pendant plusieurs dizaines de minutes à $30^{\circ} \mathrm{C}$ :

- le complexe ne se dissocie pas en $R^{*}$ et $T$;

- l'état $R^{\star}$ (caractérisé par la présence de 11-cis rétinal dans un environnement protéique lui conférant une absorption à 380 $\mathrm{nm}$, c'est-à-dire l'état Meta II) est maintenu dans $R^{*}-T$ vide, alors que dans les mêmes conditions de température une $R^{*}$ non liée à $T$ peut évoluer vers des état ultérieurs (Pfister et al., 1983). II y a donc, au niveau du site du rétinal, une stabilisation due à la liaison de $\mathrm{T}$ sur son site cytosolique (Fig. 5). 


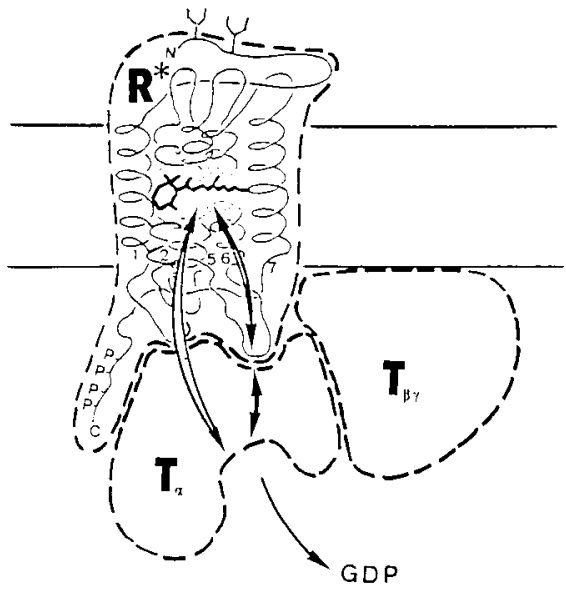

Fig. 5. Représentation imagée des interactions réciproques entre le site du rétinal, le site de liaison de la transducine, et le site nucléotidique de la transducine.

\section{Activation des protéines $G$ des sys- tèmes hormonaux}

Le parallélisme entre transduction visuelle et hormonale est tout à fait remarquable.

La protéine $G$ activée se dissocie en $\mathrm{G} \alpha \mathrm{GTP}$ et $\mathrm{G} \beta \gamma$, tout comme T (Smigel et al., 1982).

Dans le complexe RH-G formé entre le récepteur activé $(\mathrm{RH})$ et la protéine $\mathrm{G}$, l'hormone $(H)$ serait bloquée dans son site de liaison. Ceci permettrait d'interpréter pleinement les expériences de mesures d'«affinités» entre récepteur et hormones :

$R+H \underset{K_{d}}{\stackrel{K_{a}}{\rightleftharpoons}} R^{*} H \stackrel{G}{\rightleftharpoons} R^{*} H-G \stackrel{G T P}{\rightleftharpoons} R^{*} H+G$

1) en l'absence de protéine $G$, seul le premier équilibre a lieu, et l'«affinité", en réalité la constante d'équilibre de la liaison hormone-récepteur, est mesurée par le rapport $\mathrm{K}_{\mathrm{a}} / \mathrm{K}_{\mathrm{d}}$;
2) en présence de protéine $G$, mais en l'absence de tout nucléotide, on observe expérimentalement une quantité d'hormone liée au récepteur supérieure au cas a). Les auteurs parlent alors d'une "haute affinité" du récepteur pour son hormone, mais ce terme prête à confusion : en effet, le deuxième équilibre entre alors en jeu, impose à l'hormone de rester bloquée sur le récepteur, dans l'état $R^{*}-H-G$ (tout comme l'était la dissociation du rétinal de la rhodopsine); les constantes caractéristiques du premier équilibre ne sont pourtant en rien modifiées;

3) en présence de protéine $G$ et de GTP, le troisième équilibre entre en jeu à son tour. L'état $R^{\star} H$ est régénéré, et on se retrouve formellement dans le cas 1), c'està-dire la situation qualifiée de "basse affinité". Dans ce dernier cas encore, les constantes $K_{a}$ et $K_{d}$ ne sont pas modifiées. C'est la prise en compte de l'ensemble des équilibres qui explique les résultats, et non pas un "précouplage" de $G$ sur $R$ mais plutôt bien un "postcouplage" de l'hormone dans le complexe $R^{*}-H-G$.

\section{LES EFFECTEURS ENZYMATIQUES}

\section{La phosphodiestérase spécifique du GMPc}

La GMPc-phosphodiestérase (GMPc-PDE) des bâtonnets est présente à environ un exemplaire pour 100 rhodopsines. Elle est composée de 3 polypeptides ( $\alpha, \beta$ et $\eta$ ) de poids moléculaires respectifs $88 \mathrm{kDa}, 86$ $\mathrm{kDa}$ et $10 \mathrm{kDa}$. Cette enzyme possède une activité d'hydrolyse du GMPc qui est portée par le complexe PDE $\alpha \beta$. Celui-ci est maintenu dans un état inactif par PDE $\gamma$ (Hurley \& Stryer, 1982), au nombre de 2 exemplaires par holo-enzyme de PDE native (Deterre et al., 1988). Comme la transducine, la PDE est périmembranaire en milieu physiologique, mais soluble en milieu de faible force ionique. 
T $\alpha$ GTP active la PDE en agissant séquentiellement avec les sous-unités PDE $\gamma$. II semble que cette interaction conduise à la formation d'un complexe T $\alpha$-PDE $\gamma$ (Deterre et al., 1986). La PDE peut se présenter sous 3 formes : la forme PDE $\alpha \beta \gamma^{2}$, native et inactive; la forme PDE $\alpha \beta$, totalement dépourvue de ses sous-unités inhibitrices et à activité maximale; la forme intermédiaire, PDE $\alpha \beta \gamma$, dont l'activité semble intermédiaire mais reste à déterminer avec exactitude.

Une activation tout à fait artificielle de la PDE par protéolyse trypsique a été observée, et 3 mêmes états PDE $\alpha \beta \gamma^{2}$ PDE $\alpha \beta \gamma$ et PDE $\alpha \beta$ de la PDE ont été isolés (Deterre et al., 1988).

\section{L'adénylate cyclase}

C'est une glycoprotéine transmembranaire activée par la sous-unité GoGTP, et par protéolyse trypsique limitée. Bien qu'elle ait une spécificité tout à fait distincte de celle de la PDE, un mécanisme d'activation similaire, par levée d'inhibition, pourrait être envisagé.

\section{ACTIVATION ARTIFICIELLE DES PRO- TÉINES G PAR LES IONS FLUOROALU- MINATES}

\section{Activation de l'adénylate cyclase}

Dans la description de la stimulation de l'adénylate cyclase par les ions fluorures (de $\mathrm{Na}$ ou de K) il a été très vite clair que l'activation par les hormones et l'activation par les fluorures présentaient des caractéristiques différentes : site d'action membranaire pour les hormones, intracellulaire pour les ions fluorures. En isolant la protine de régulation de la cyclase (la protéine G), Pfeuffer (1977) a montré que cette protéine, indispensable à l'activation de la cyclase par le GTP, était également le site d'action des ions fluorures.

Sternweiss \& Gilman, en 1982, ont pu montrer la nécessité de la présence de traces d'aluminium dans le milieu pour que l'action des ions fluorures soit observable. II est suggéré, mais non prouvé, que l'espèce active était l'ion AlF $_{4}$ agissant directement sur la protéine $\mathrm{G}$.

\section{Activation de la phosphodiestérase}

Elle était observée dès 1977 par Sitaramayya et al. (1977) et décrite par Stein et al. (1985) comme résultant de l'activation de la transducine par les ions fluorures.

L'activation de $T$ par les ions fluorures, mesurée soit par la capacité de T d'activer la phosphodiestérase, soit par les changements de conformation de $\mathrm{T}$ (solubilité, profils de protéolyse trypsique) a montré (Bigay et al., 1985, 1987) que :

- le fluorure est actif sous forme d'ion $\mathrm{AlF}_{4}$;

- le site d'action de $\mathrm{AlF}_{4}$ est la transducine, et plus précisément sa sous-unité $\alpha$.

- l'activation de la transducine nécessite la présence d'un GDP dans le site nucléotidique. Une interaction directe a lieu entre l'ion fluoroaluminate et l'oxygène libre du $\beta$-phosphate du GDP. L'ensemble GDP$\mathrm{AlF}_{4}$ mime, dans le site nucléotidique, la présence d'un GTP (Fig. 6). 


\section{LES SECONDS MESSAGERS}

Le plus anciennement connu est sans conteste I'AMPcyclique, comme rappelé plus haut. Depuis, d'autres molécules ont été décrites comme tenant ce rôle : le GMPcyclique (comme dans le cas de la vision), les ions $\mathrm{Ca}^{++}$(comme dans les phénomènes de contraction musculaire), le diacylglycérol et l'inositol triphosphate (produits par activation de la phospholipase $\mathrm{C}$ et la dégradation des phosphatidylinositols).

Les effets de ces seconds messagers peuvent être classés en 2 catégories, selon qu'ils agissent

- directement, comme dans le cas de la vision, où la liaison de GMPc aux canaux de la membrane plasmique contrôle la conductivité de ces canaux;

- indirectement, comme dans le cas de l'adénylate cyclase, où la modulation de concentration intracellulaire d'AMPc contrôle l'activité de kinases, qui à leur tour contrôlent par phosphorylation des protéines responsables de l'activité cellulaire.
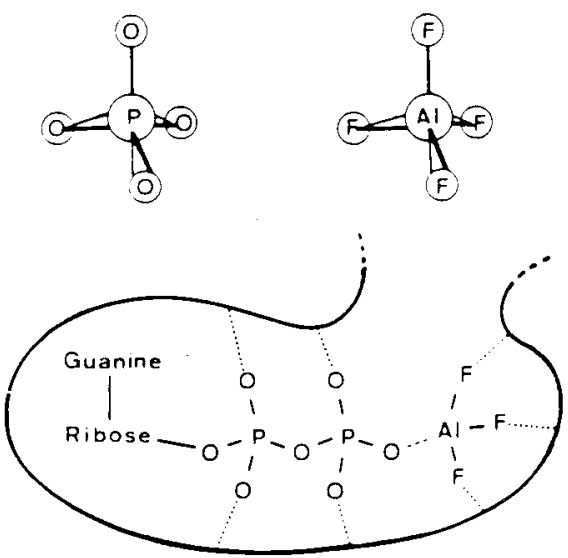

Fig. 6. Analogies structurales entre l'ion $\mathrm{PO}_{4}^{-}$et l'ion $\mathrm{AlF}_{4}^{-}$. Mode d'action de l'ion $\mathrm{AlF}_{\overline{4}}$ dans le site nucléotidique d'une protéine $\mathrm{G}$.

\section{CONCLUSION}

La transmission de signal utilisant les seconds messagers est un processus très répandu. Des évidences de plus en plus nombreuses montrent les analogies très profondes entre les espèces protéiques mises en jeu : récepteurs, protéines $G$, effecteurs enzymatiques, et entre les caractéristiques des cascades enzymatiques respectives.

On connaît à présent le rôle des sousunités $\alpha$ des protéines de couplage. Cependant, le rôle des sous-unités $\beta$ et $\gamma$ reste obscur. II doit cependant être capital, à en juger par l'exceptionnelle conservation des séquences polypeptidiques correspondantes dans tous les systèmes (Manning \& Gilman, 1983). Les mécanismes d'arrêt ou d'inactivation de ces cascades enzymatiques ne sont que partiellement connus. On a décrit le rôle de la phosphorylation du récepteur par une kinase (Liebman \& Pugh, 1980; Wilden et al., 1986; Bennett et Sitaramayya, 1988), le rôle d'une protéine supplémentaire (l'arrestine) pour inactiver le récepteur (Wilden et al., 1986). On connaît la voie d'inactivation de la protéine de couplage qui hydrolyse son GTP par une activité GTPasique intrinsèque à sa sous-unité $\alpha$ (Godchaux et Zimmerman, 1979; Kühn, 1980).

Cependant, une cellule qui possède toute une panoplie de récepteurs, de protéines $G$ et d'effecteurs, et subit l'effet de toute une gamme d'hormones diverses, doit effectuer les aiguillages correspondants à sa nécessité vitale. Neer \& Clapham (1988) suggèrent qu'il existerait une localisation subcellulaire des protéines des cascades transductionnefies, permettant l'interaction spécifique entre le récepteur, sa protéine G associée et l'effecteur correspondant. 


\section{RÉFÉRENCES}

Applebury M.L. \& Hargrave P.A. (1986) Molecular biologie of the visual pigments. Vision Res. 26, 1881-1895

Bennett N., Michel-Villaz M. \& Kühn H. (1982) Light-induced interaction between rhodopsin and the GTP-binding protein. Meta rhodopsin II is the major photoproduct involved. Eur. J. Biochem. 27, 97-103

Bennett N. \& Sitaramayya A. (1988) Inactivation of photoexcited rhodopsin in retinal rods : the roles of rhodopsin kinase and $48 \mathrm{k}$-Da protein (Arrestin). Biochemistry 27, 1710-1715

Bigay J., Deterre P., Pfister C. \& Chabre M. (1985) Fluoroaluminates activate transducinGDP by mimicking the $\gamma$-phosphate of GTP in its binding site. Fed. Eur. Biochem. Soc. Lett. 191. 181-185

Bigay J., Deterre P., Pfister C. \& Chabre M. (1987) Fluoride complexes of aluminium and beryllium act on G-proteins as reversibly bound analogues of the $\gamma$-phosphate of GTP. Eur. Mol. Biol. Organ. J. 6, 2907-2913

Chabre M. (1985) Molecular mechanisms of visual phototransduction in retinal rod cell. Ann. Rev. Biophys. Chem. 14, 331-360

Chabre M., Pfister C., Deterre P. \& Kühn H. (1984) The mechanism of control of cGMP phosphodiesterase by photoexcited rhodopsin in retinal cells. Analogies with hormone controlled systems. In : Hormones and Cell Regulation. (J.E. Dumont \& J. Nuñez, ed.) 8, 87-97. Elsevier Science Publishers, BV

Chabre M., Bigay J., Bruckert F., Bornancin F., Deterre P., Pfister C. \& Vuong T.M. (1988) Visual signal transduction : the cycle of transducin shuttling between rhodopsin and cGMP phosphodiesterase. In : Symposia on quantitative Biology. Vol. 53, 313-324 : Molecular Biology of Signal Transduction. Cold Spring Harbor Laboratories

Deterre P., Bigay J., Robert M., Pfister C., Kühn H. \& Chabre M. (1986) Activation of retinal rod cyclic GMP-phosphodiesterase by transducin : characterization of the complex formed by phosphodiesterase inhibitor and transducin $\alpha$ subunit. Proteins Struct. Funct. Genet. 1, 188193

Deterre P., Bigay J., Forquet F., Robert M. \& Chabre M. (1988) The cGMP phosphodieste- rase of retinal rods is regulated by two inhibitory subunits. Proc. Natl Acad. Sci. USA 85, 24242428

Dixon R.A.F., Sigal I.S., Rands E., Register R.B., Candelore M.R., Blake A.D. \& Strader C.D. (1987) Ligand binding to the $\beta$-adrenergic receptor involves its rhodopsin-like core. Nature 326, $73-77$

Dohlman H.G., Caron M.G. \& Lefkowitz R.J. (1987) A family of receptors coupled to guanine nucleotide regulatory proteins. Biochemistry 26 , 2657-2664

Dolphin A. (1987) Nucleotide binding proteins in signal transduction and disease. Trends Neurosci. 10, 53-57

Fesenko E.E., Kolesnikov S.S. \& Lyubarsky A.L. (1985) Induction by cGMP of cationic conductance in plasma membrane of retinal rod outer segments. Nature 313, 310-313

Gilman A.G. (1987) G proteins : transducers of receptor-generated signals. Ann. Rev. Biochem. $56,615-649$

Godchaux III W. \& Zimmerman W.F. (1979) Membrane-dependant guanine nucleotide binding and GTPase activities of a soluble protein from bovine rod cell outer segments. J. Biol. Chem. 254, 7874-7884

Graziano M.P. \& Gilman A.G. (1987) Guanine nucleotide-binding regulatory proteins : mediators of transmembrane signaling. Trends Pharmacol. Sci. 81, 478-481

Hargrave P.A., McDowell J.H., Curtis D.R., Wang J.K., Juszczak E., Fong S.L., Rao J.K.M. \& Argos P. (1983) The structure of bovine rhodopsin. Biophys. Struct. Mech. 9, 235-244

Hurley J.B. \& Stryer L. (1982) Purification and characterization of the $\alpha$-subunit of the cyclic GMP phosphodiesterase from retinal rod outer segments. J. Biol. Chem. 257, 11094-11099

lyengar R. \& Birnbaumer L. (1987) Signal transduction by G-proteins. ISI Atlas of Science : Pharmacology, 213-331

Kühn H. (1980) Light- and GTP-regulated interactions of GTPase and other proteins with bovine photoreceptor membranes. Nature 283, 587-589

Liebman P.A. \& Pugh E.N. (1980) ATP mediates rapid reversal of cGMP phosphodiesterase activation in visual receptor membranes. Nature $237,734-736$ 
Limbird L.E. \& Lefkowitz R.J. (1977) Resolution of $\beta$-adrenergic receptor binding and adenylate cyclase activity by gel exclusion chromatography. J. Biol. Chem. 252, 799-802

Manning D.R. \& Gilman A.G. (1983) The regulatory components of adenylate cyclase and transducin : a family of structurally homologous guanine nucleotide binding proteins. J. Biol. Chem. 268, 7059-7063

Miller R.J. (1988) G-proteins flex their muscles. Trends Neurosci. 11, 3-6

Neer E.J. \& Clapham D.E. (1988) Roles of Gprotein subunits in transmembrane signalling. Nature 333, 129-134

Ovchinnikov U.A. (1982) Rhodopsin and bacteriorhodopsin : structure-function relationships. Fed. Eur. Biochem. Soc. Lett. 148, 179-191

Owen W.G. (1986) Tionic conductances in rod photoreceptors. Ann. Rev. Physiol. 49, 743-764

Pfeuffer R. (1977) GTP-binding proteins in membranes and the control of adenylate cyclase activity. J. Biol. Chem. 252, 7224-7234

Pfister C., Kühn H. \& Chabre M. (1983) Interaction between photoexcited rhodopsin and peripheral enzymes in retinal rods. Influence on the post metarhodopsin II decay and phosphorylation rate of rhodopsin. Eur. J. Biochem. 136, 489-499

Proud C.G. (1986) Guanine nucleotides, protein phosphorylation and the control of translation. Trends Biochem. Sci. 11, 73-77

Rodbell M., Birnbaumer L. \& Pohl S.I. (1971) The glucagon-sensitive adenylate cyclase system in plasma membrane of rat liver. V. An obligatory role of guanyl nucleotides in glucagon action. J. Biol. Chem. 246, 1877-1882
Sitaramayya A., Virmaux N. \& Mandel P.I. (1977) On the mechanism of light activation of retinal rod outer segment cyclic GMP phosphodiesterase. Light activation : influence of bleached rhodopsin and KF-DE inhibition. Exp. Eye Res. 25, 163-169

Smigel M.D., Northup J.K. \& Gilman A.G. (1982) Characteristics of the guanine nucleotidebinding regulatory component of adenylate cyclase. Recent Progr. Horm. Res. 38, 601-622

Spiegel A.M. (1987) Signal transduction by nucleotide binding proteins. Mol. Cell. Endocrinol. 49, 1-16

Stein P.J., Halliday K.R. \& Rasennick M.M. (1985) Photoreceptor GTP-binding protein mediates fluoride activation of PDE. J. Biol. Chem. $260,9081-9084$

Sternweiss P.C. \& Gilman A.G. (1982) Aluminium : a requirement for activation of the regulatory component of adenylate cyclase by fluoride. Proc. Natl Acad. Sci. USA 79, 4888-4891

Strader C.D., Sigal I.S., Candelore M.R., Rands E., Hill W.S. \& Dixon R.A.F. (1988) Conserved aspartic acid residues 79 and 113 of the $B$ adrenergic receptor have different roles in receptors function. J. Biol. Chem. 263, 1026710271

Stryer L. \& Bourne H. (1986) G proteins : a family of signal transducers. Annu. Rev. Cell Biol. 2, 391-419

Wilden U., Hall S.W. \& Kühn (1986) Phosphodiesterase activation by photoexcited rhodopsin is quenched when rhodopsin is phosphorylated and binds the intrinsic 48-kDa protein of rod outer segments. Proc. Natl Acad. Sci. USA 83, 1174-1178 